\title{
MODELING OF A COAXIAL LIQUID OXYGEN / GASEOUS HYDROGEN INJECTION ELEMENT UNDER HIGH-FREQUENCY ACOUSTIC DISTURBANCES
}

\author{
S. Beinke ${ }^{1,2}$, D. Banuti ${ }^{3}$, J. Hardi $^{1}$, M. Oschwald ${ }^{1}$, \\ and B. Dally ${ }^{2}$ \\ ${ }^{1}$ Institute of Space Propulsion \\ German Aerospace Center (DLR) \\ Lampoldshausen, Hardthausen D-74239, Germany \\ ${ }^{2}$ School of Mechanical Engineering \\ The University of Adelaide \\ Adelaide 5005, South Australia, Australia \\ ${ }^{3}$ Institute for Aerodynamics and Flow Technology \\ German Aerospace Center (DLR) \\ Göttingen, Göttingen 37073, Germany
}

\begin{abstract}
An experimental combustor, designated BKH, is operated at DLR Lampoldshausen to investigate high-frequency combustion instability phenomena. The combustor operates with liquid oxygen (LOx) and gaseous or liquid hydrogen propellants at supercritical conditions analogous to real rocket engines. An externally imposed acoustic disturbance interacts with a series of 5 coaxial injection elements in the center of the chamber. A combination of experimental analysis and numerical modeling is used to provide further insight and understanding of the $\mathrm{BKH}$ experiments. Optical data from the BKH experiments are analyzed to extract the response of the flame at the excitation frequency. A new method for reconstructing the acoustic field inside the chamber from dynamic pressure sensor data is used to describe the evolution of the acoustic mode and the local disturbance in the flame zone. An Unsteady Reynolds-Averaged Navier-Stokes (URANS) model of a single $\mathrm{BKH}$ injection element subjected to representative transverse acoustic velocity excitation has been computed using a specialized release of the DLR TAU code. The single-element model reproduces the retraction of the dense LOx core during transverse velocity excitation as observed experimentally. The model also provides further insight into the flattening and flapping of the flame. The flapping is identified as the oxygen core being transported by the transverse acoustic velocity.
\end{abstract}

(C) The Authors, published by EDP Sciences. This is an open access article distributed under the terms of the Creative Commons Attribution License 4.0 (http://creativecommons.org/licenses/by/4.0/). 


\section{INTRODUCTION}

High-frequency combustion instabilities refer to a coupling between acoustic and combustion processes which occurs inside combustion chambers. If unimpeded, this coupling may produce acoustic disturbances that can affect the operation and structural integrity of the combustion chamber. High-frequency combustion instabilities are not yet fully understood and cannot be predicted, increasing risk and necessitating extensive ground testing to verify the safe operation of new hardware designs and configurations. Central to the problem of combustion instabilities is how a flame responds to an acoustic disturbance in order to support the instability.

Various experimental combustion chambers have been developed to study combustion instability phenomena using subscale hardware. The combustion instability experiments feature additional diagnostics and instrumentation to observe the response of a flame to either natural or externally imposed disturbances. The data from such experiments may also be used to validate combustion instability models. Once validated, the models may be used to provide further insight into the experiments themselves and eventually as a tool for predicting combustion instabilities.

This work compares the results of a simplified modeling approach with experimental data collected from a high-frequency combustion instability experiment, designated BKH, operated at the DLR Institute of Space Propulsion. The current work aims to model the response of the reacting flow within the BKH combustor to an externally imposed acoustic disturbance. In this paper, a brief background of the $\mathrm{BKH}$ combustor is first provided. A method for reconstructing the acoustic field from pressure sensor data is then described. Optical data from BKH experiments showing the response of the flame to the applied acoustic field are then presented and analyzed to produce results for comparison with numerical data. The results of a single-element computational fluid dynamics (CFD) model under representative acoustic forcing are compared with the experimental data.

\section{EXPERIMENTAL SETUP}

The BKH combustor operates at pressures ranging from 40 (subcritical oxygen pressure) to 60 bar (supercritical oxygen pressure) using cryogenic LOx and hydrogen $\left(\mathrm{H}_{2}\right)$ propellants. The combustion chamber features a rectangular geometry with windows located on each side for optical access to the near-injector region, and a secondary nozzle and toothed wheel for excitation. The combustion chamber volume is $240 \mathrm{~mm}$ long, $50 \mathrm{~mm}$ wide, and $200 \mathrm{~mm}$ high, not including the main and secondary nozzles. A series of five coaxial study elements are 


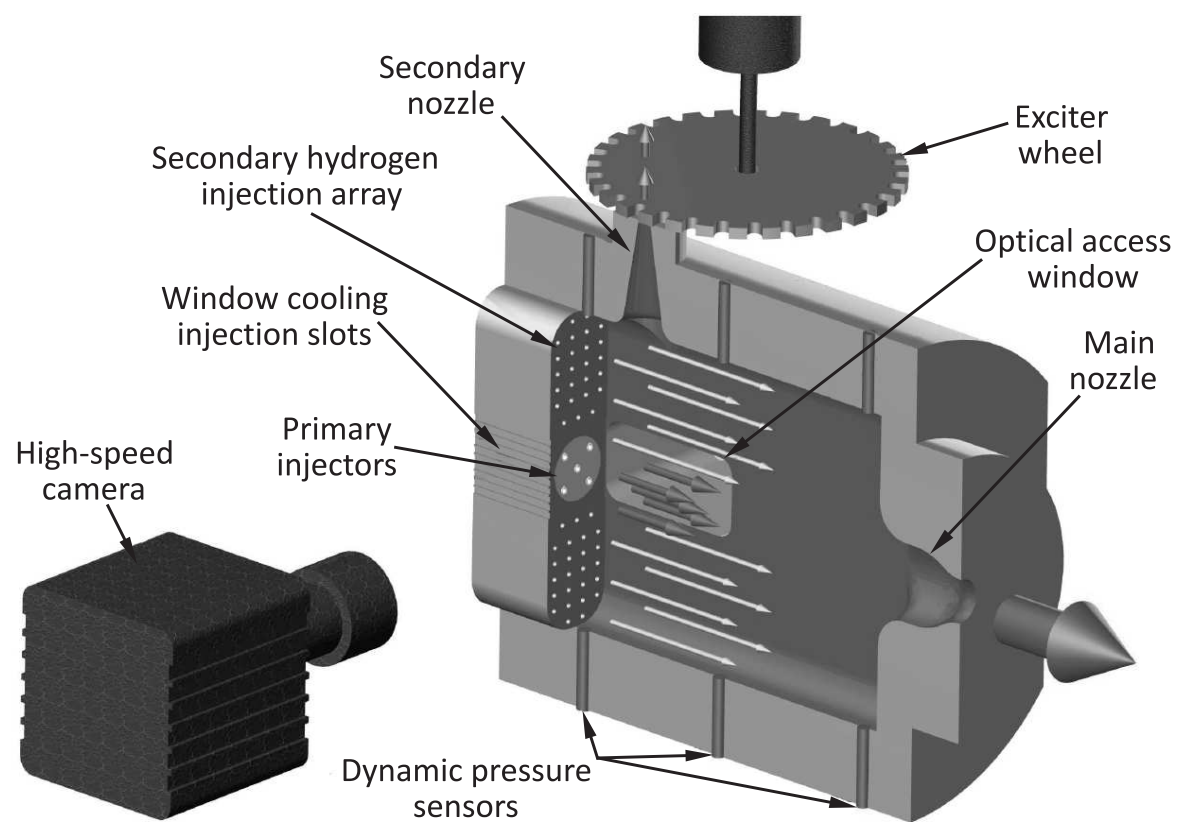

Figure 1 Concept diagram of the BKH combustor

positioned in a matrix pattern in the middle of the chamber. Figure 1 depicts the BKH chamber configuration.

During operation, the toothed wheel is rotated at a controlled speed in order to periodically interrupt the flow through the secondary nozzle. The periodic interruption of flow causes an acoustic disturbance to propagate back into the chamber. The frequency of the disturbance is controlled by the rotational speed of the wheel. Dynamic pressure measurements are recorded at various locations on the combustion chamber walls as shown in Fig. 2 to resolve the acoustic field.

The response of the study elements to the acoustic disturbance is observed via high-speed shadowgraph and $\mathrm{OH}$ chemiluminescence $\left(\mathrm{OH}^{*}\right)$ imaging captured through the windows in the sides of the chamber. The rectangular shaped internal volume was designed to deliberately fix the orientation of the transverse acoustic mode. The dimensions of the chamber are such that the resonant mode frequencies of the chamber volume match those of full-scale upper-stage rocket engines. The BKH experiments are conducted at the European Research and Technology test facility P8 for cryogenic rocket engines at DLR Lampoldshausen. Additional information on BKH can be found in [1-3].

During BKH experiments, the excitation frequency is steadily ramped so that it passes through the resonant modes of the combustor. Each resonant mode has 


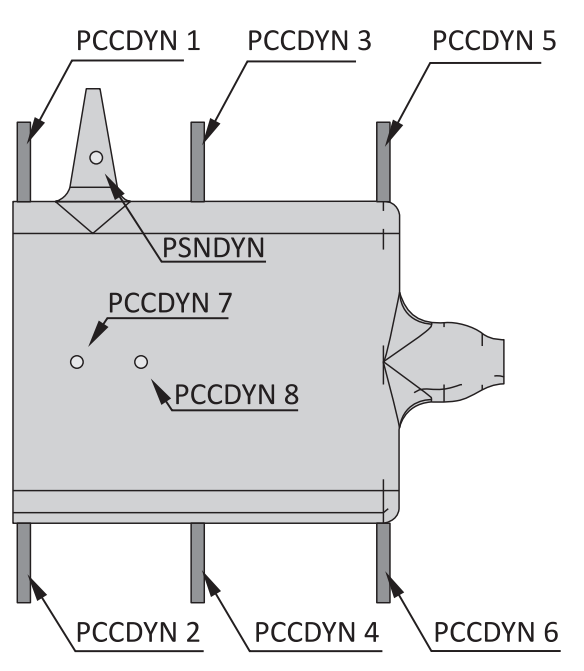

Figure 2 Dynamic pressure sensor positions in the BKH combustor a different orientation and distribution which produces a different disturbance around the study elements. By controlling which mode is excited using the excitation system, the response of the flame to different acoustic disturbances can be observed. When the first longitudinal (1L) mode is excited, the study elements lie in a pressure antinode. This means that the local acoustic fluctuation around the study elements is primarily an acoustic pressure fluctuation. Similarly, during transverse mode excitation, the study elements lie near a pressure nodal line and are subjected to acoustic velocity fluctuations. By selecting the mode frequency, the response of the study elements to either acoustic pressure or acoustic velocity can be observed.

\section{EXPERIMENTAL DATA}

Experimental data have been collected from BKH experiments operated at the 60-bar ROF (ratio of oxidizer to fuel) 6 operating point. Dynamic pressure sensor data are used to reconstruct the acoustic disturbance acting upon the study elements. High-speed optical images of the flame zone are also collected for comparison and validation of numerical results. This section begins by presenting the method used to reconstruct the acoustic field from dynamic pressure sensor data. The optical data that have been collected is then described. To provide a better comparison with numerical results, the mean response of the flame at the excitation frequency is extracted using Dynamic Mode Decomposition (DMD) analysis of the optical data.

\subsection{Dynamic Pressure Sensor Data}

In [4], the acoustic modes within the BKH combustor have been identified by comparing the relative phase and amplitude of dynamic pressure data from different sensor positions. An improved method that involves interpolation of the complex acoustic amplitude over a representative domain has since been developed. This new method allows the structure and phase of the instantaneous 
acoustic field to be visualized and shows how the acoustic field evolves with increasing excitation frequency during BKH experiments.

To reconstruct the acoustic field, it is assumed that the acoustic disturbance takes the form of a harmonic disturbance: $\hat{A} e^{i \hat{\omega} t}$ where $\hat{A}$ is the complex amplitude; $\hat{\omega}$ is the complex frequency; and $t$ is the time. The complex amplitude of a field consists of a real and imaginary components at each position in the field:

$$
\hat{A}_{(x, y, z)}=A_{R(x, y, z)}+A_{I(x, y, z)} i .
$$

To reconstruct the acoustic field, the complex amplitude at each sensor position is determined and interpolated to estimate the acoustic field. To determine the complex amplitude at each sensor position, a sample of dynamic pressure sensor data, $p^{\prime}$, is processed. The dynamic pressure signal is first band pass filtered about the excitation frequency to extract the monofrequent sinusoidal disturbance. The two components of the complex amplitude are then determined using the following two relations:

$$
\begin{array}{r}
|\hat{A}|=\left|A_{R}+A_{I} i\right|=\sqrt{\frac{\sum_{1}^{n} p_{n}^{\prime 2}}{n}} \cdot \sqrt{2}=p_{\mathrm{RMS}}^{\prime} \cdot \sqrt{2} \\
\operatorname{angle}(\hat{A})=\operatorname{angle}\left(A_{R}+A_{I} i\right)=\operatorname{phase}\left(p^{\prime}\right)
\end{array}
$$

Equation (1) states that the maximum amplitude of the signal can be reasonably approximated as $\sqrt{2}$ times the root mean square value for a harmonic disturbance. Equation (2) relates the phase angle of the complex amplitude with the phase of the monofrequent signal. For reconstruction purposes, the phase is only required to be known relative to all other sensor positions. For this work, the phase of each filtered dynamic pressure sensor signal was normalized against the phase of the PCCDYN 2 sensor signal shown in Fig. 2.

Figure 3 shows how the acoustic amplitude and phase at each sensor position evolve over a single BKH test. During the test, the excitation frequency is ramped from 2 to $5 \mathrm{kHz}$, passing through the 1L and first transverse (1T) modes. The $1 \mathrm{~L}$ mode occurs at approximately $18.5 \mathrm{~s}$ into the test. It is marked by the sensors at opposite ends of the combustor being out of phase and sensors 3 and 4 , located in the middle of the combustor, have a low amplitude. The $1 \mathrm{~T}$ mode occurs at approximately $32 \mathrm{~s}$ when sensors on the top and bottom walls of the chamber are out of phase and sensors 7 and 8, located in the middle of the chamber, have a low amplitude.

The real and complex parts of the amplitude are calculated from Eqs. (1) and (2). These values are then interpolated over an area representing the combustor volume. As the dynamic pressure sensor data has been previously filtered to the excitation frequency, it is assumed that the disturbance is monofrequent and should take the form of an acoustic eigenmode with no sharp discontinuities. 


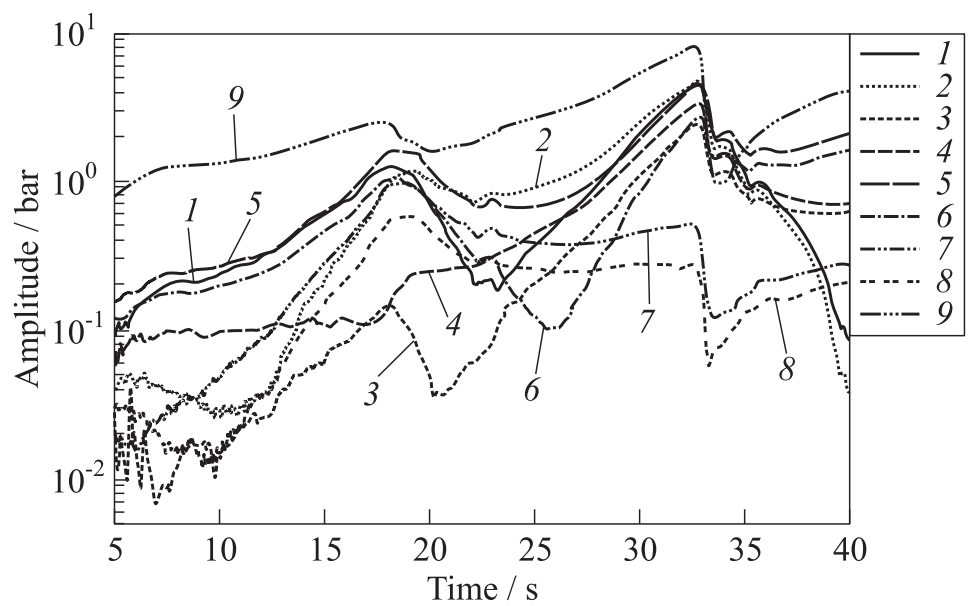

(a)

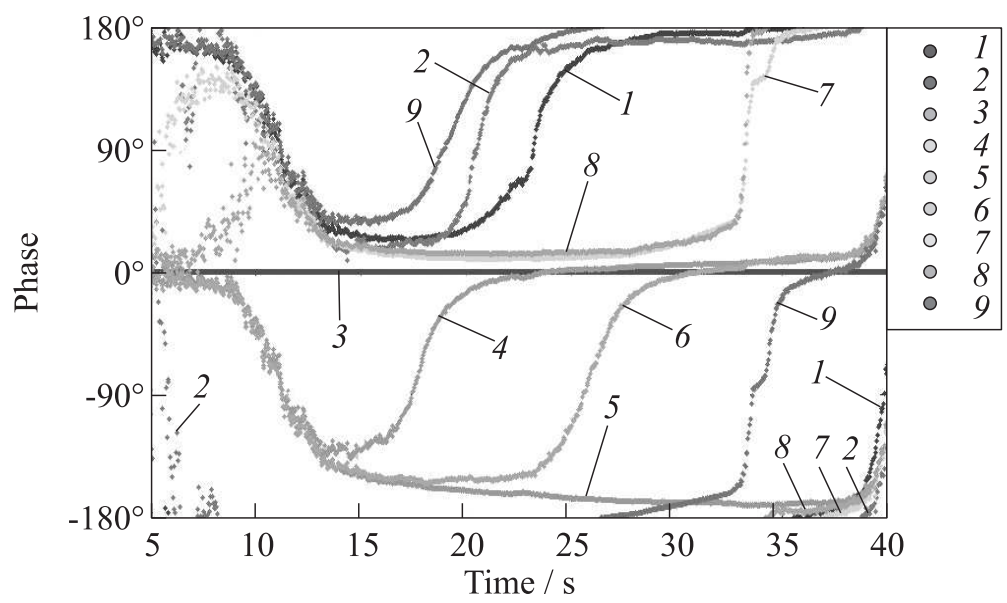

(b)

Figure 3 Amplitude $(a)$ and phase $(b)$ of the acoustic disturbance at each sensor position vs. test time: $1-$ PCCDYN $1 ; 2-\operatorname{PCCDYN~} 2 ; 3-\operatorname{PCCDYN~} 3 ; 4-$

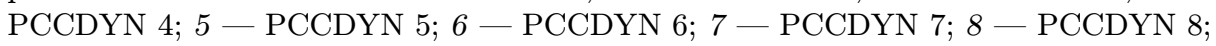
and $9-\mathrm{PSNDYN}$

A spline interpolation scheme is therefore used to interpolate the complex amplitude between sensor positions. The interpolated result can then be modulated at the excitation frequency to animate the reconstructed acoustic field. Snapshots of this reconstruction are shown in Fig. 4.

Identification of the peak $1 \mathrm{~L}$ and $1 \mathrm{~T}$ mode distributions is of particular interest. The reconstruction during peak $1 \mathrm{~L}$ and $1 \mathrm{~T}$ mode excitation are shown 

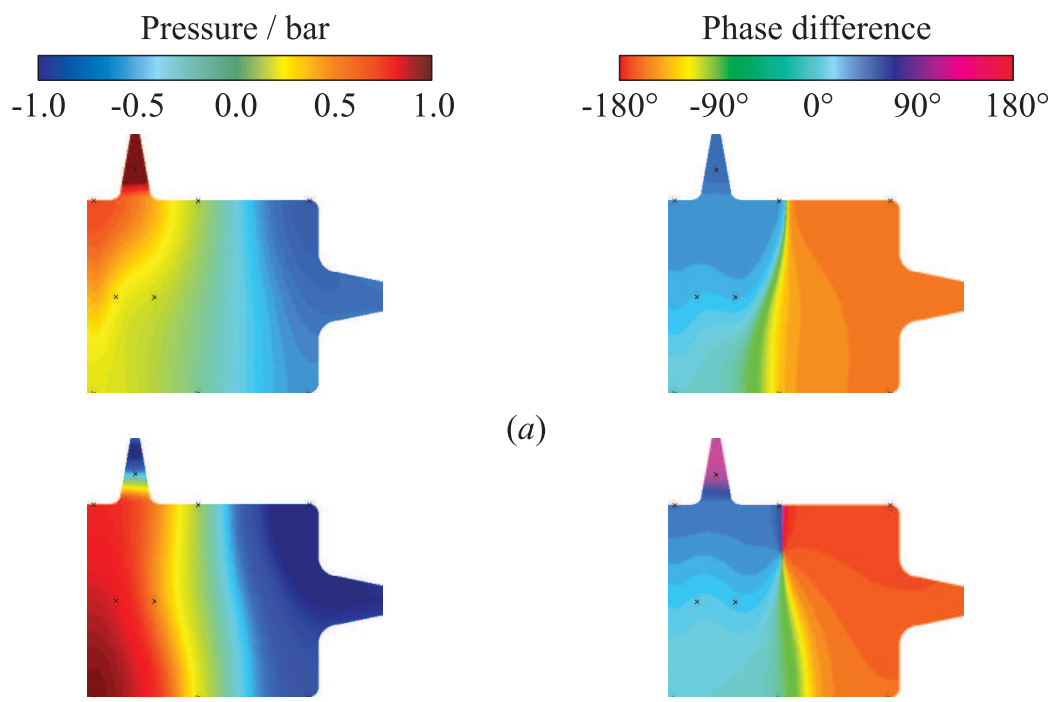

(a)
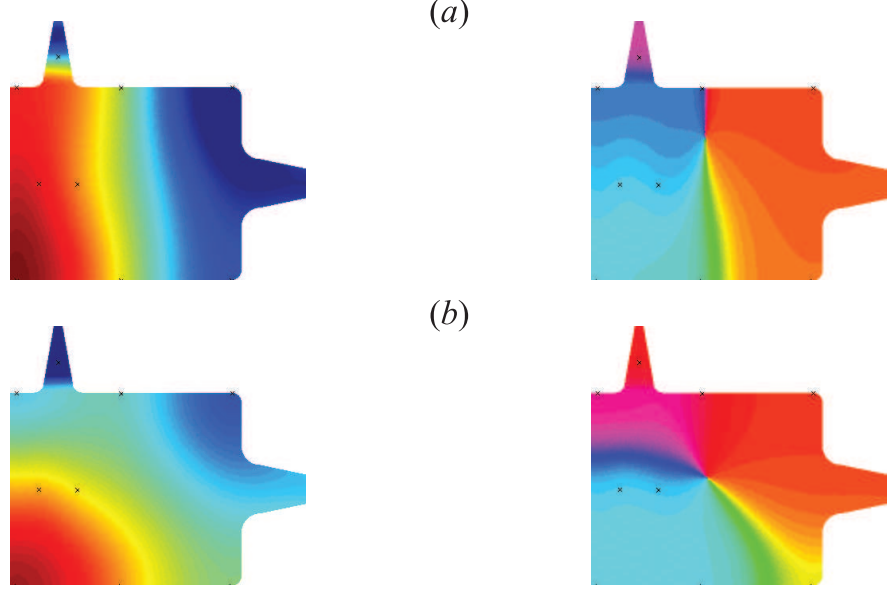

(b)
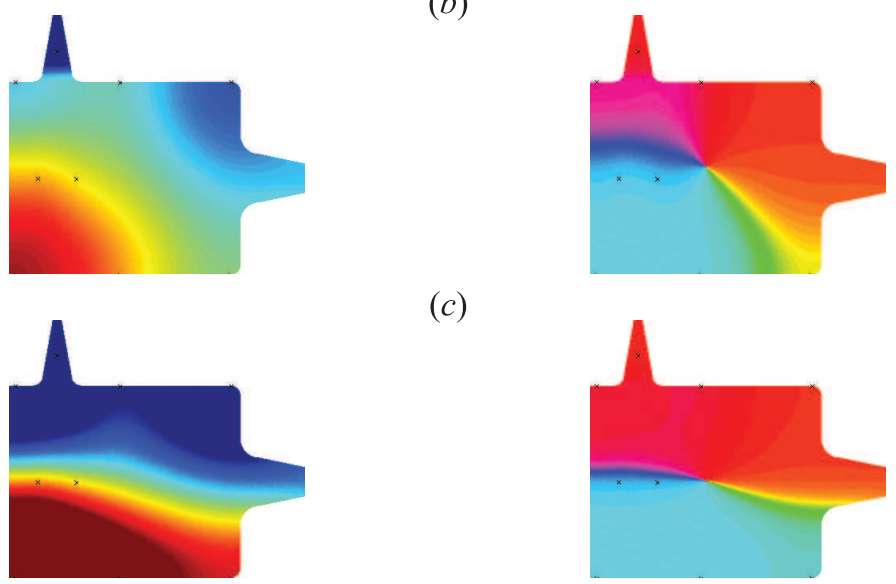

(c)
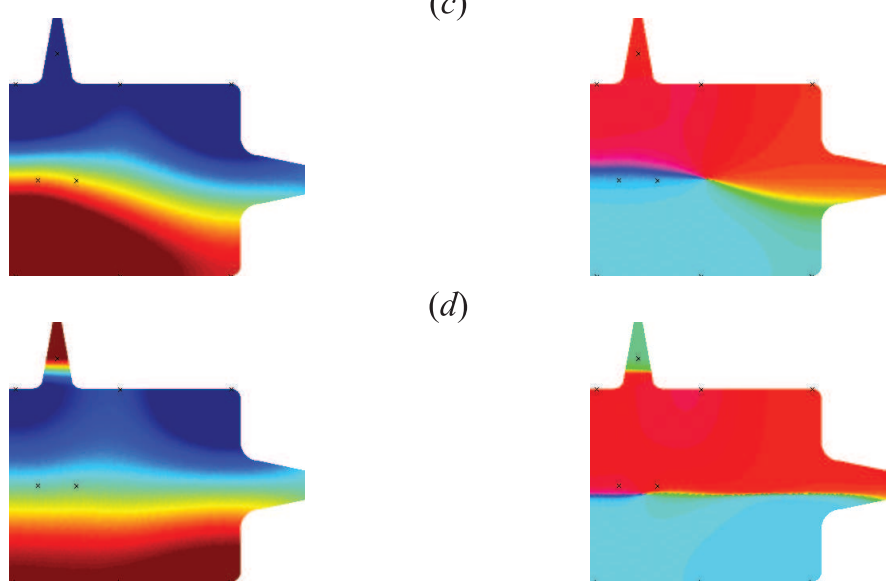

(d)

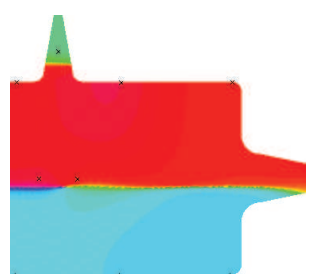

(e)

Figure 4 Reconstructed acoustic field for different test periods: $(a) t=15 \mathrm{~s}$; $(b) 20$; (c) 25; (d) 30; and (e) $t=35 \mathrm{~s}$ 


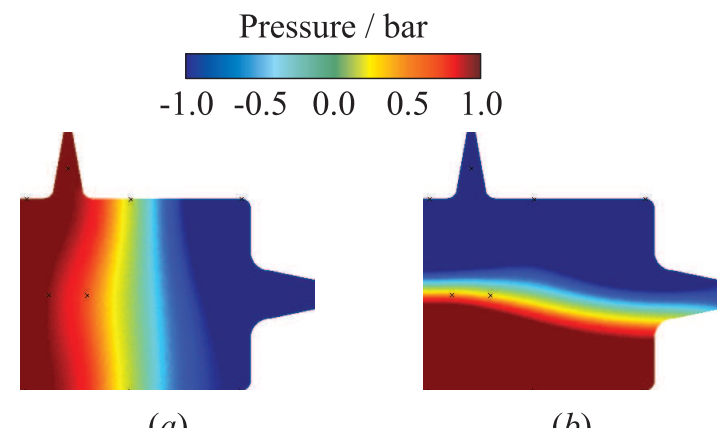

(a)

(b)

Figure 5 Reconstructed resonant modes: (a) $1 \mathrm{~L}$ mode peak amplitude at $t=18 \mathrm{~s}$; and $(b) 1 \mathrm{~T}$ mode peak amplitude at $t=32 \mathrm{~s}$

in Fig. 5. The acoustic disturbance acting locally upon the study elements by each acoustic mode can then be extracted from the reconstructed acoustic field. Knowledge of acoustic disturbance local to the injection elements is used to determine representative numerical disturbances for modeling the $\mathrm{BKH}$ experiments.

\subsection{Optical Data}

High-speed $\mathrm{OH}$ chemiluminescence and shadowgraph images have been collected at the 60-bar ROF 6 operating point. Line-of-sight images are recorded through windows in the side walls of the chamber as shown in Fig. 6. Due to the pentainjector configuration, two of the five injection elements are hidden behind other elements and only three distinct jets are visible. The optical setup employed during the BKH experiments has been previously described in $[2,5]$.

Samples of the original optical datasets are shown in Figs. 6 and $7 a$. Figure 6 shows samples from a test period when the excitation frequency does not match a resonant mode of the combustor volume. Figure $7 a$ shows samples from a test period when the excitation frequency matches the $1 \mathrm{~T}$ mode of the combustion chamber volume and Fig. $7 b$ shows time-averaged images from a period of $1 \mathrm{~T}$ excitation.

As stated in section 2, during transverse excitation, the acoustic velocity acts across the injection plane. The acoustic velocity disturbs and displaces the dense LOx core as it fluctuates across the injection elements. The acoustic motion also increases mixing of oxidizer and fuel streams and increases shedding of the dense LOx core. The length of the flame is observed to shorten and retract towards the faceplate during $1 \mathrm{~T}$ mode excitation as shown in Fig. $7 a$ (left column).

The experimental optical data show a variety of unsteady turbulent motion. To provide a better comparison with numerical results, the optical data are 


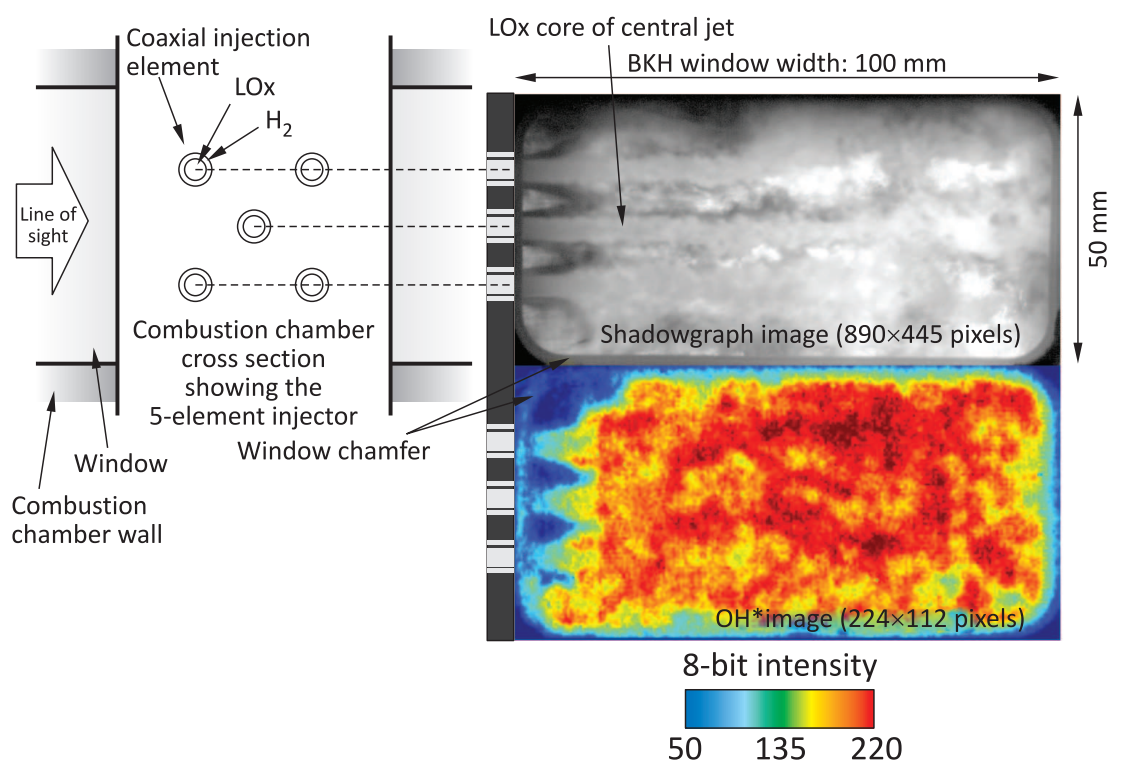

Figure 6 Optical data during off-resonance excitation

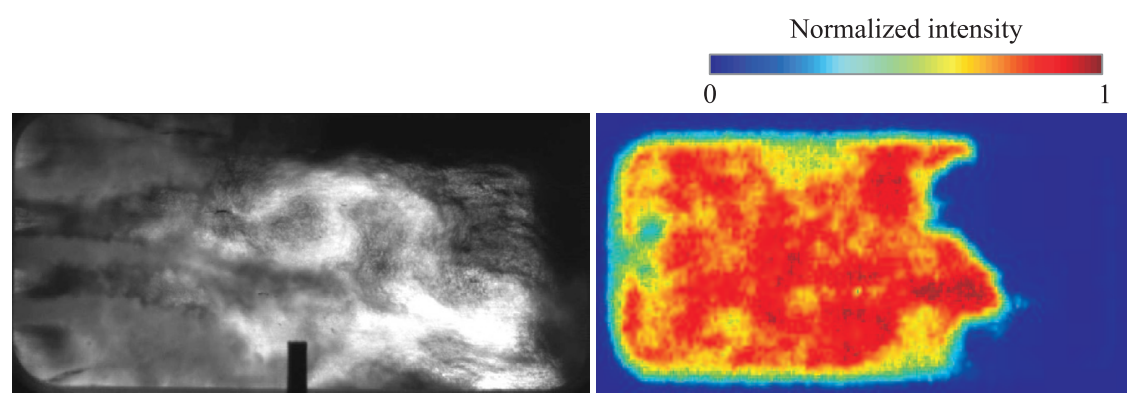

(a)
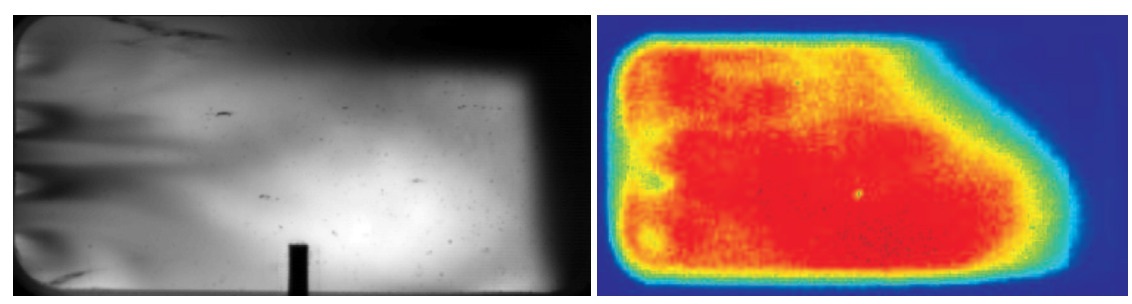

(b)

Figure 7 Optical data $(a)$ and mean optical data $(b)$ during $1 \mathrm{~T}$ mode excitation: left column - shadowgraph; and right column - $\mathrm{OH}$ chemiluminescence 
processed to extract the mean response of the flame to the imposed acoustic disturbance. Dynamic mode decomposition is described by Schmid [6] and is useful for identifying and sorting coherent fluctuations of data at different frequencies. Dynamic mode decomposition is often applied to study periodically fluctuating phenomena and has been previously used in combustion instability research in order to detect coherent fluctuations of flame visualization data $[7,8]$.

Shadowgraph and $\mathrm{OH}$ chemiluminescence images have been captured from a test period with acoustic excitation tuned at the chamber $1 \mathrm{~T}$ mode frequency. Sequences of $n=1000$ images were used to calculate DMD modes. Each mode has a temporal component describing how it fluctuates in time and a spatial component representing the fluctuation of each pixel about its mean value. The mean image from each sample is shown in Fig. $7 b$. The relative contribution of each DMD mode to the original imaging data was considered by computing the $\ell_{2}$ norm, $|\phi|$, of the temporal component as in $|\phi|=\sqrt{\sum_{k=1}^{n}\left|\phi_{k}\right|^{2}}$.

Figure 8 plots the $\ell_{2}$ norm vs. mode frequency for each of the shadowgraph and $\mathrm{OH}$ image sequences. The spectra for the shadowgraph data (Fig. $8 a$ ) shows two distinct peaks at the excitation frequency and its first overtone. The spectra for the $\mathrm{OH}$ chemiluminescence data (Fig. 8b) shows the same two peaks and a number of other additional peaks. These additional peaks have been identified as an artefact from the high-speed camera and are not of interest to the present work.

The modes corresponding to the excitation frequency and its overtone are selected for further analysis. The real part of the spatial components of the selected modes are shown in Fig. 9. The shadowgraph DMD modes (Figs. $9 a$ and $9 b$ ) show the fluctuation of the dense LOx core. By comparing the shadowgraph DMD modes with the mean image (see Fig. $7 b$, left column), a striped structure is visible in the region marked "A." This region is near the end of

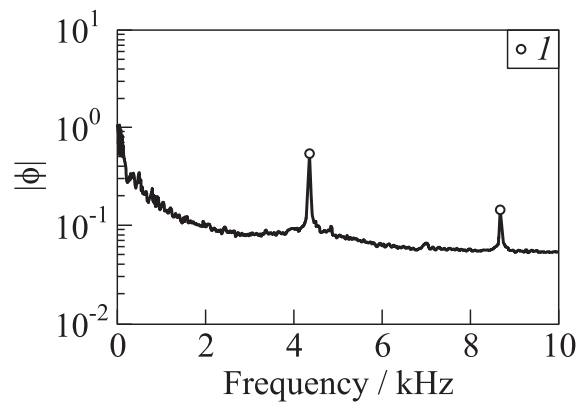

(a)

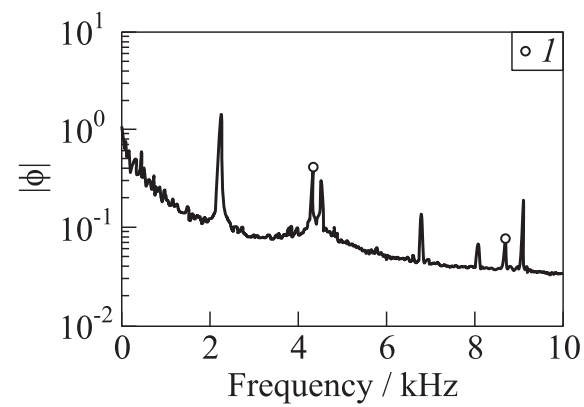

(b)

Figure 8 The $\ell 2$ norm vs. frequency of processed optical samples: $(a)$ shadowgraph sample; (b) $\mathrm{OH}$ chemiluminesence sample; and 1 - selected modes 


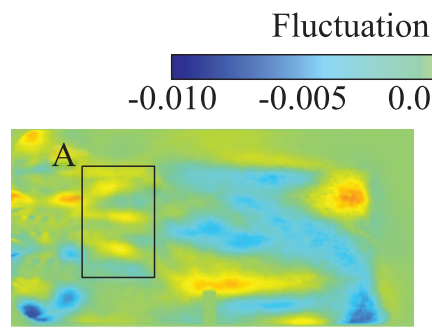

(a)

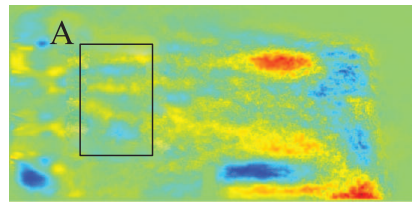

(b)

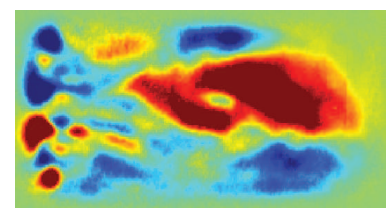

(c)

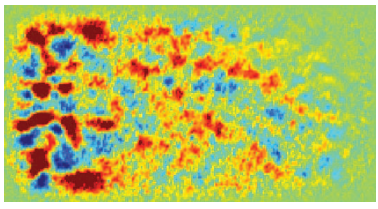

(d)

Figure 9 Spatial components of the selected DMD modes: (a) real part of shadowgraph mode at the excitation frequency $4344 \mathrm{~Hz} ;(b)$ real part of shadowgraph mode at the first overtone of the excitation frequency $8689 \mathrm{~Hz} ;(c)$ real part of $\mathrm{OH}$ mode at the excitation frequency $4348 \mathrm{~Hz}$; and $(d)$ real part of $\mathrm{OH}$ mode at the first overtone of the excitation frequency $8697 \mathrm{~Hz}$

the retracted oxygen core of the central injection element indicating a uniform vertical motion of the oxygen core at the excitation frequency.

The $\mathrm{OH}$ DMD mode at the excitation frequency (see Fig. 9c) shows similar horizontal structures as the shadowgraph modes in the same region indicating that the region of highest $\mathrm{OH}$ intensity follows the oxygen core. The $\mathrm{OH}$ mode at the overtone of the excitation frequency (see Fig. 9d) appears to exhibit similar structures on the left side of the image in the near-injector region. The downstream part of all DMD modes show large structures which are attributed to turbulence and the bulk motion of the flame moving in and out of the window region.

The selected DMD modes highlight the response of the flame at the excitation frequency and its overtone. The vertically alternating pattern of horizontal structures observed in the selected DMD modes highlight the motion of the flame at the excitation frequency. The flame is forced upwards and downwards by the acoustic velocity acting vertically across the injection plane. As these structures are visible in shadowgraph images, it suggests that the oxygen core is being transported as a horizontal structure by the acoustic velocity. The flame surface then follows the motion of the oxygen core.

The DMD analysis has been used to identify and describe the mean motion of the flame during transverse excitation. The identified phenomena will be compared with the results of the numerical model described in the following sections. 


\section{NUMERICAL METHOD}

To investigate the response of the BKH study elements to an imposed acoustic disturbance, a model of a single injector under acoustic forcing has been developed. The model consists of steady and unsteady computations using a specialized version of the DLR TAU code for modeling real gas properties $[9,10]$. A steady-state Reynolds-averaged Navier-Stokes (RANS) computation is used to model the single injector under steady-state operation without acoustic forcing. Unsteady RANS is used to model the mean response of the flame to an imposed acoustic disturbance for comparison with the fluctuations identified in subsection 3.2. During the unsteady computation, the boundary conditions of the domain are modified to impose an acoustic disturbance representative of the local acoustic fluctuation around an injection element in the BKH combustor experiments. The DLR Tau code, numerical domain, grid, and excitation method used are described in the following subsections.

\subsection{Computational Fluid Dynamics Solver}

The DLR TAU code is a hybrid (structured/unstructured) grid Godunov-type finite-volume flow solver for the compressible Euler and Navier-Stokes equations. Second-order spatial accuracy is reached by a MUSCL (Monotone Upstream Scheme for Conservation Laws) reconstruction. The TAU code has been validated for a range of steady and unsteady flow cases $[11,12]$.

Cryogenic oxygen at high pressures, as encountered in rocket engines, behaves differently than ideal gases. To account for these real gas effects, a new real gas mixture model has been developed and implemented in the DLR TAU code. The model and its validation are described in more depth in $[9,10]$.

As the chamber pressure of the experiment considered in this work is above the supercritical pressure of all of the injected species, liquid and gaseous phases no longer exist and thus need not be distinguished. In this new model, cryogenic oxygen is treated as an Eulerian continuum. Real gas properties are computed from the high fidelity modified Benedict-Webb-Rubin (MBWR) equation of state (EoS) of Younglove [13] and stored in a library during a preprocessing step. Thermodynamic state variables, such as pressure, enthalpy, heat capacities, speed of sound, etc., are all computed consistently from the real-gas EoS. Realgas corrections to the transport coefficients are evaluated following Lemmon and Jacobsen [14]. Here, an ideal-gas equation is solved for each of the species $\mathrm{H}_{2}$, $\mathrm{OH}, \mathrm{H}_{2} \mathrm{O}, \mathrm{H}_{2} \mathrm{O}_{2}, \mathrm{O}, \mathrm{H}$, and $\mathrm{HO}_{2}$, and the real gas MBWR library is used for $\mathrm{O}_{2}$.

For the current work, the one-equation Spalart-Allmaras [15] turbulence model was used. The Spalart-Allmaras model is a relatively robust turbulence model with good convergence that can be applied to a variety of flows. It was selected for this work due to its simplicity and ease of implementation. 
The Spalart-Allmaras model is known to overpredict the spreading rate when modeling round-jet free-shear flows but captures mixing layer flows well $[16,17]$. A MAPS+ Riemann solver is used to handle low Mach numbers and high-density gradients. Stabilization, if necessary for the computation of high-density ratio shear layers, is carried out by reducing the spatial order to 1.95. In this way, the numerical damping is minimally increased while mass, momentum, and energy remain conserved.

In the current work, a finite rate chemistry scheme that solves the Arrhenius equation is used. The Jachimowski mechanism is described in [18] and involves 8 species and 17 reactions. Under the conditions encountered in high-pressure hydrogen-oxygen combustion, the reaction can be considered to reach equilibrium and turbulent mixing constitutes the rate determining process. Lempke et al. [19] studied the difference between laminar and turbulent chemistry in a similar configuration and found virtually no difference in the temperature field. For this reason, an explicit turbulent combustion model is not used in the current work. Turbulence combustion interaction is implicitly included by the addition of a turbulent contribution to the laminar diffusion coefficient and thermal conductivity for each species. This approach is described by Karl [12] and has been used previously to compute oxygen-hydrogen combustion [20].

To perform unsteady computations, a Jameson-type dual time stepping scheme with a physical time step size of $5.0 \mu$ s was used for unsteady calculations. Two hundred inner iterations were computed for each physical time step and a reduced CFL (Courant-Friedrichs-Lewy) number of 0.2 was used.

\subsection{Domain and Boundary Conditions}

The domain used for the CFD calculations is shown in Fig. 10. The domain is three-dimensional (3D) in order to study the asymmetric flow field produced by acoustic velocity fluctuations and includes many details of the oxygen feed system. The oxygen post was modeled in detail to investigate oxygen feed system coupling which has been identified as a possible feedback mechanism in recent combustion instability experiments [21]. Only a short section of the hydrogen feed system before the injection plane is included. Dirichlet boundary conditions are used to specify the flow rates into the domain. The injection conditions and mass flow rates are specified to match the 60-bar, ROF 6 experimental operating point.

An unstructured mesh with approximately 600,000 points was used for this work. A mesh independence study showed that this mesh produced the same results as a much finer mesh with 1.4 million points. The coarser mesh was used to reduce the computational requirements of the unsteady computations. A structured boundary layer was prescribed for each physical wall boundary condition while tetrahedra were prescribed at other boundaries and to fill the internal volume. The mesh was refined near the tip of the oxygen post to resolve 


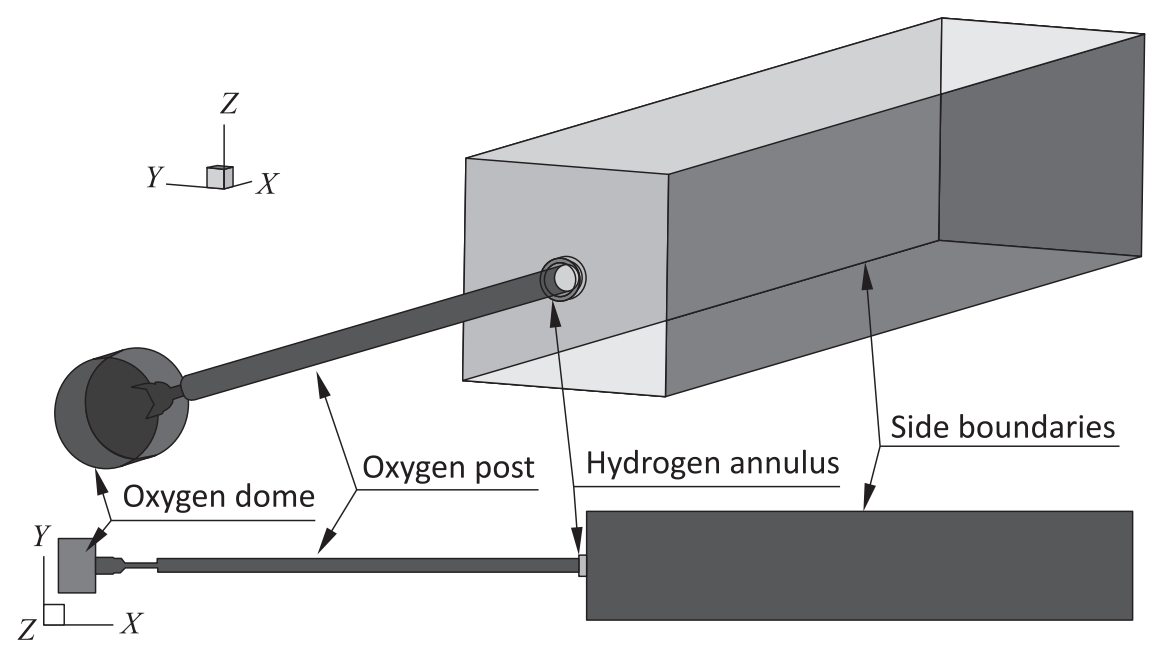

Figure 10 Domain used for single injector computations.

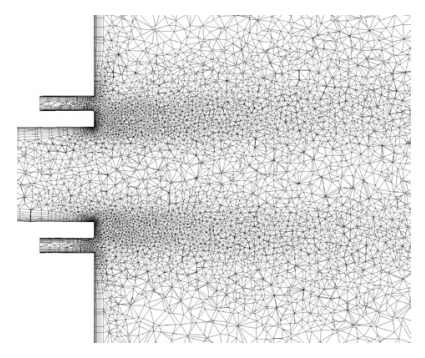

(a)

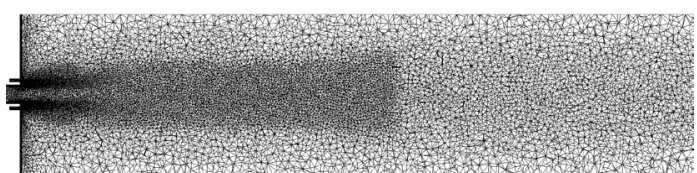

(b)

Figure 11 Mesh used for CFD computations: $(a)$ refinement near the injection plane; and $(b)$ refinement in the flame zone

the shear layer between the oxygen and hydrogen streams. The mesh was also refined throughout the flame zone directly downstream of the injector. A slice through the center of the mesh is shown in Fig. 11.

A box extending from the injection plane is used to represent the combustion chamber volume. During an initial steady-state computation, the side boundaries of the box are treated as inviscid walls. The inviscid wall condition ensures there is no mass flow through the side boundaries and that the flame is contained within the domain. The end boundary of the box, opposite the injection plane, is treated as a near-field boundary condition. The near-field boundary condition does not represent a physical wall but rather internal fluid boundaries. 
A state is specified at the near-field boundary and the flux across the boundary is determined by the difference between this state and the interior points in the numerical domain. This boundary condition was used as it allowed a fluid state at a specified pressure to be prescribed along a large portion of the boundary of the numerical domain effectively setting the mean pressure across the domain. The near-field boundaries were prescribed to match the experimental combustion chamber pressure.

For unsteady computations, the side walls are also changed to a representative near-field boundary condition. The flow contained within the domain by ensuring the imposed state at the side boundaries matches the inviscid wall solution. In order to impose an acoustic disturbance, the state of the near-field boundaries is modified after each physical time step. An acoustic disturbance representative of a transverse acoustic mode is imposed by prescribing a pressure gradient and velocity disturbance to vertically opposite sides of the numerical domain.

The near-field boundaries were chosen due to the relative ease of implementation with existing numerical tools. However, there are a number of consequences to this approach. For the current work, the state at all of the near-field boundaries was defined as a pure $\mathrm{H}_{2}$ environment. Therefore, the current model is analogous to a single element surrounded by a pure $\mathrm{H}_{2}$ environment. However, in the BKH experiment and full-scale systems, each element is in close proximity to other neighboring coaxial elements. The pure $\mathrm{H}_{2}$ environment means that all flow exiting the domain through a side boundary is replaced by a pure $\mathrm{H}_{2}$ mixture entering the domain from the opposite boundary. The additional $\mathrm{H}_{2}$ affects the total ROF of the domain. Nevertheless, as most of the heat release occurs near the injection plane and the dimensions of the domain have been chosen to ensure the flame does not come into direct contact with the near-field boundaries, the models are useful for studying the response of the flame to an imposed acoustic disturbance.

\section{NUMERICAL RESULTS}

In this section, the results of the CFD computations with the DLR TAU code are presented. A steady-state solution representing an unexcited flame was first computed. The response of the single injector to an imposed transverse acoustic velocity disturbance was then modeled by subjecting the steady-state solution to a representative disturbance during an unsteady computation.

\subsection{Steady-State Solution}

A RANS solution was computed to provide a steady-state result which would then be excited by imposing an acoustic disturbance via fluctuating boundary conditions. Figure 12 shows the result of this computation. 


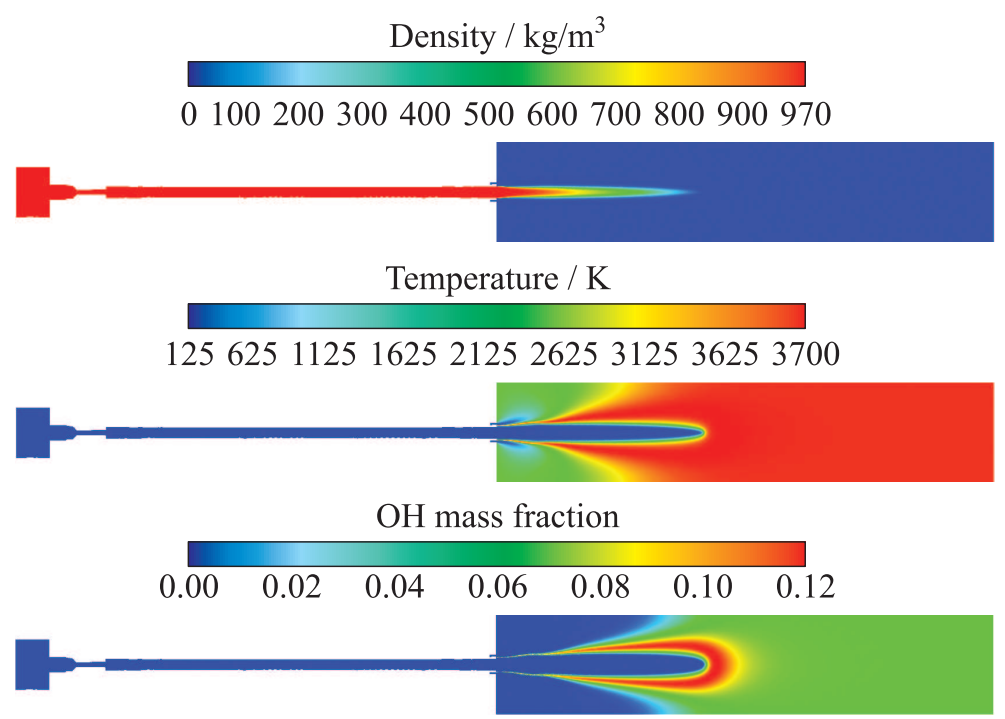

Figure 12 Cut through center plane of steady-state result

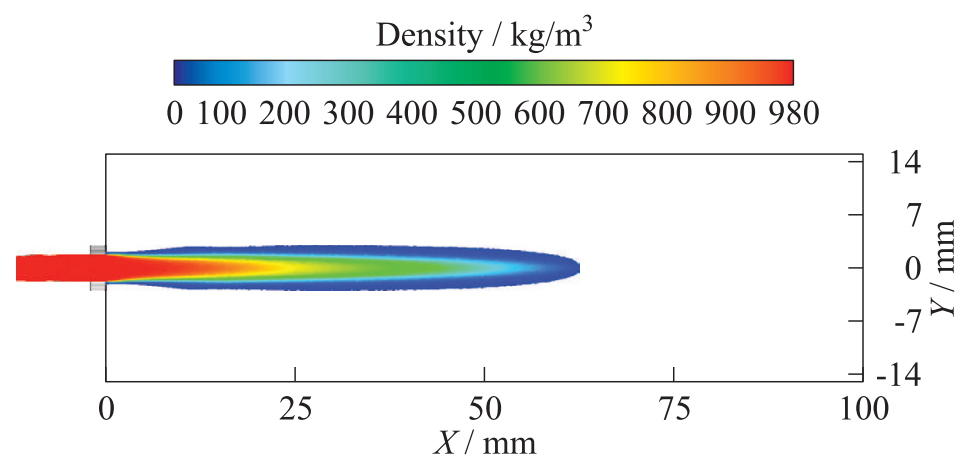

Figure 13 The 2D plot of steady-state density distribution (cutoff at density $10 \mathrm{~kg} / \mathrm{m}^{3}$ )

The CFD results were postprocessed in order to compare them with the available experimental optical data. Experimental optical access is through $100 \times 50 \mathrm{~mm}$ windows in the side of the chamber which view the area directly downstream of the injection plane as shown in Fig. 6. To compare with the collected optical data, the $3 \mathrm{D}$ flow field is postprocessed to produce representative two-dimensional (2D) images. 
The shadowgraph images show primarily the location of the dense LOx core, a 2D plot of density is shown for comparison in Fig. 13. The RANS computation produces a dense LOx core which is approximately $62 \mathrm{~mm}$ long. This length is based on an arbitrary density cutoff value of $10 \mathrm{~kg} / \mathrm{m}^{3}$. The experimental optical data suggest a slightly longer liquid core which sometimes extends the length of the window $(100 \mathrm{~mm})$. However, the length of the core fluctuates considerably as large parts at the end of the liquid core break off. This phenomena is not captured in the steady-state result. The shorter core in the numerical results could also be due to the domain not being representative of the $\mathrm{BKH}$ flow field where neighboring jets would produce a fuel-lean environment and lengthen the liquid core.

\subsection{Transverse Velocity Excitation}

The single injector model was subjected to a fluctuating disturbance representative of a transverse instability. The disturbance was designed using the results of the acoustic field reconstruction described in subsection 3.1. The reconstruction assumes linear acoustics and indicates that during $1 \mathrm{~T}$ mode excitation, the central injection element lies along the pressure nodal line and in a velocity antinode. To represent this disturbance, the pressure and the velocity at the boundaries of the domain were modulated to impose a representative transverse acoustic velocity across the domain.

A plot of the resultant velocity fluctuation in the vertical direction is shown in Fig. 14. The amplitude of the disturbance approximately matches that predicted by the modal reconstruction. The disturbance was applied uniformly along the length of the domain. The frequency of the dis-

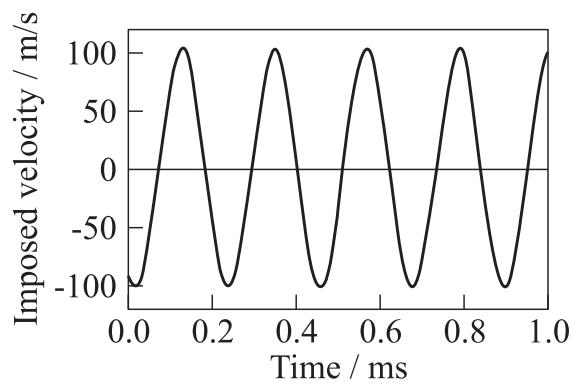

Figure 14 Transverse velocity disturbance imposed upon single injector model turbance was chosen to be $4400 \mathrm{~Hz}$ which is approximately the $1 \mathrm{~T}$ mode frequency of the BKH combustor. The imposed velocity fluctuation caused the flame to fluctuate up and down as expected. The jet is observed to fluctuate at the same frequency as the excitation frequency.

Figure 15 shows different snapshots of the oxygen core as excitation is imposed. Figure $15 a$ shows the initially undisturbed oxygen core. As the disturbance is imposed, the core is observed to flatten in the direction normal to the acting acoustic velocity and form a sheet-like structure as shown in Fig. 15b. The center of the sheet directly downstream from the injector contains a dense oxygen 

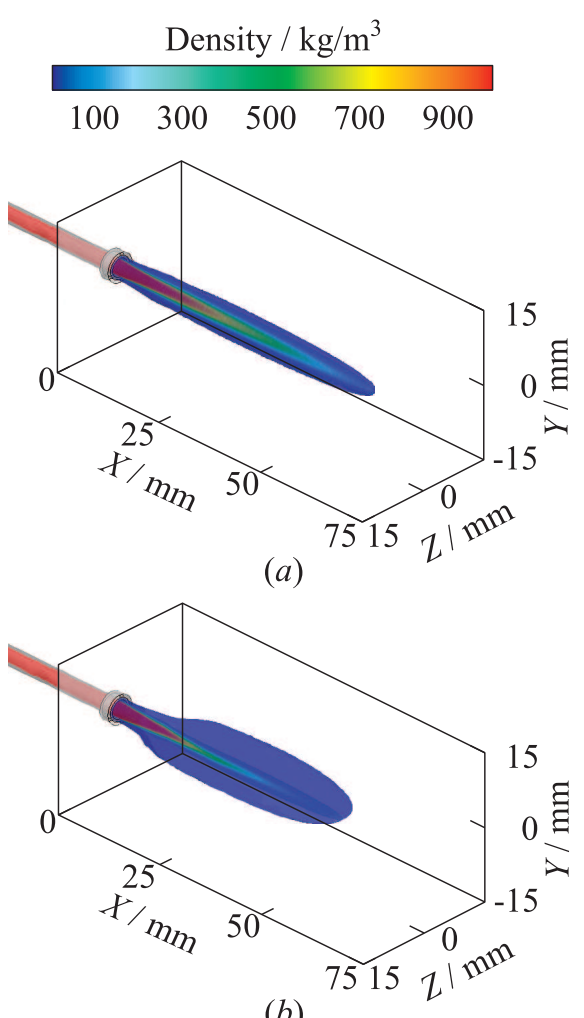

(b)

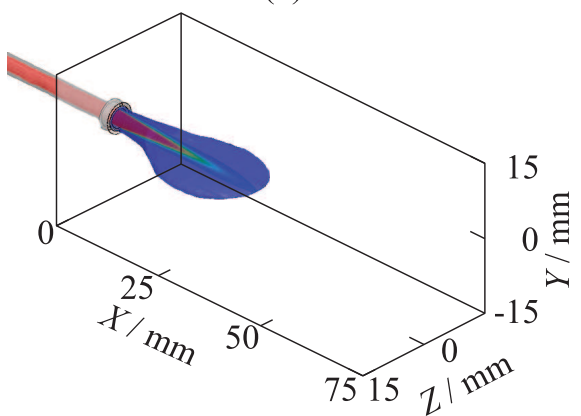

(c)

Figure 15 Change in dense oxygen core after acoustic excitation is imposed (cutoff at density $10 \mathrm{~kg} / \mathrm{m}^{3}$ ): (a) unperturbed oxygen core; $(b)$ flattening of the core after excitation is imposed; and $(c)$ after retraction has completed core and is not greatly displaced by the acoustic disturbance. The outer parts of the sheet, which are not as dense as the core, are more readily perturbed by the imposed acoustic velocity and traverse a considerable distance per acoustic cycle. The difference in displacement between the center and sides of the sheet causes the sheet to appear to "flap." As more continuous acoustic forcing is imposed, the jet begins to retract towards the injection plane. After approximately 15 acoustic cycles, the jet does not retract any further and maintains a consistent length (Fig. 15c). The shortened core continues to exhibit a flattened sheetlike structure downstream and fluctuates at the excitation frequency. The displacement of the retracted core is shown in Fig. 16a. A side view of the retracted oxygen core is shown in Fig. 16b. The retracted core has a length of approximately $34 \mathrm{~mm}$ using the same density cutoff value of $10 \mathrm{~kg} / \mathrm{m}^{3}$ as in the steady-state case.

\section{DISCUSSION}

Modeling of rocket engine injectors under transverse acoustic excitation has been conducted by various other research groups using largeeddy simulation (LES) [22, 23] and URANS [24]. Flattening, flapping, and retraction of the liquid oxidizer core from a coaxial injection element under transverse acoustic excitation have previously been identified in 


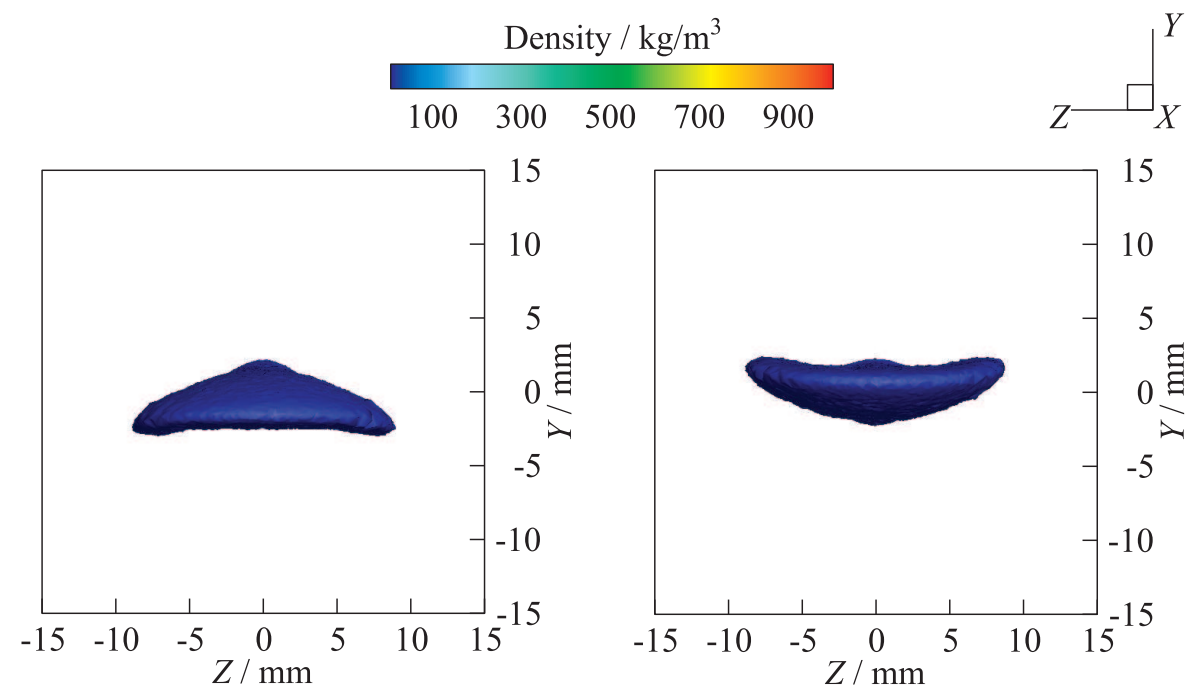

(a)

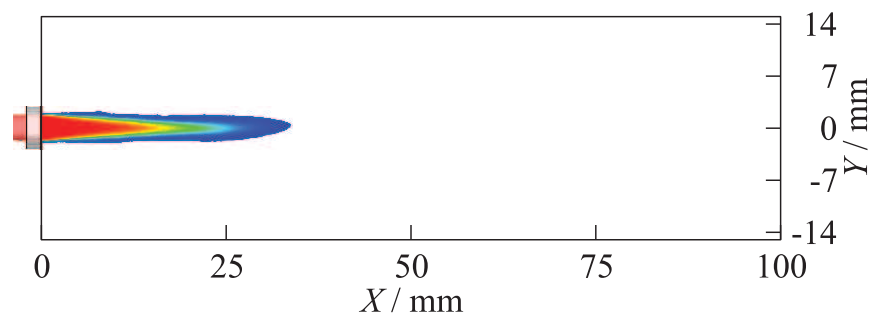

(b)

Figure 16 Snapshots of retracted liquid core during acoustic cycle viewed from downstream $(a)$ and 2D plot of density distribution after core has retracted (b) (cutoff at density $10 \mathrm{~kg} / \mathrm{m}^{3}$ )

methane flames using LES [22]. The numerical and experimental results presented in this work show the same phenomena for an oxygen-hydrogen flame.

Hardi et al. [5] have previously reported how the dense LOx core from a coaxial injector retracts towards the injection plane for different amplitudes of transverse acoustic excitation. The results of this analysis suggested that under high acoustic amplitudes, the core retracts to an $L / D$ ratio (ratio of length to diameter of oxygen inlet) of approximately 10 . The numerical results exhibited a similar retraction in the oxygen core length. Using the aforementioned $10 \mathrm{~kg} / \mathrm{m}^{3}$ cutoff value to define the edge of the oxygen core leads to a retracted core $L / D$ ratio of 9.7 . 
The numerical results provide further insight into the experimental data. The flapping of the oxygen core, which is associated with a similar flapping of the flame surface, is not immediately recognizable from the line-of-sight optical images. The interpretation of the horizontal structures identified by the DMD analyis (see Figs. $9 a$ and $9 b$, region A) is assisted by the numerical results. Improved methods of postprocessing numerical results for comparison with experimental data will facilitate a better understanding of the experimental data.

While considerable agreement was found between the numerical and experimental results, the numerical model has a number of limitations. As only a single injector is modeled, the influence of neighboring elements is not included. The neighboring injection elements in the $\mathrm{BKH}$ experiment act to shield the central study element from the hydrogen-rich flow directly above and below, thereby determining which chemical species are transported into the flame. The imposed numerical disturbance effectively introduces additional fuel into the numerical domain which then mixes with the flame. As the numerical model mixes with fresh hydrogen instead of combustion products from neighboring injection elements, the numerical results are believed to overpredict the breakup of the jet.

\section{CONCLUDING REMARKS}

An analysis and comparison of experimental optical and dynamic pressure sensor datasets with a model of a single injection element under representative forcing has been presented. The complimentary analysis of experimental and numerical results has facilitated further understanding and interpretation of experimental observations.

A method for reconstructing the acoustic field from experimental pressure sensor data has been introduced. The reconstruction was used to describe the evolution of the acoustic field with increasing acoustic excitation frequency and to determine the localized acoustic disturbance acting upon the flame during excitation of the $1 \mathrm{~T}$ mode. The DMD analysis of optical data from BKH experiments has identified fluctuating structures and features in the flame zone which match the acoustic excitation frequency. The transverse acoustic velocity acting locally upon the flame during $1 \mathrm{~T}$ mode excitation was found to force the flame transversally. The comparison of structures identified in the DMD analysis of shadowgraph and $\mathrm{OH}$ images suggests that the flame follows the position of the oxygen core which is being transported as a horizontal structure by the acoustic velocity.

For the first time, it has been shown that the simplified model of a single injection element subjected to an acoustic disturbance representative of a $1 \mathrm{~T}$ mode reproduces key experimental observations such as the retraction of the dense oxygen core and flapping of the outer core surface. The additional insight from the numerical model has allowed the structures identified through the DMD analysis to be interpreted as the flapping of the flame. 


\section{ACKNOWLEDGMENTS}

This work is conducted within the scope of the DLR Pro-Tau project. The authors would like to thank Bernd Wagner, Sebastian Karl, Volker Hannemann, and the members of the Aerodynamics and Flow Technology group at DLR Göttingen for the continued support and expertise provided. The authors would also like to thank Hideto Kawashima from JAXA for his contribution to the development of the acoustic reconstruction method and Matthew Wierman from Purdue University for instruction on DMD analysis.

Research has been assisted with a grant from the Sir Ross and Sir Keith Smith Fund (Smith Fund) (www.smithfund.org.au). The support is acknowledged and greatly appreciated. The Smith Fund by providing funding for this project does not verify the accuracy of any findings or any representations contained in it. The Smith fund does not accept any responsibility or liability from any person, company, or entity that may have relied on any written report or representations contained in this report if that person, company, or entity suffers any loss (financial or otherwise) as a result.

\section{REFERENCES}

1. Hardi, J., M. Oschwald, and B. Dally. 2011. Flame response to acoustic excitation in a rectangular rocket combustor with $\mathrm{LOx} / \mathrm{H}_{2}$ propellants. CEAS Space J. 2:4149.

2. Hardi, J. S. 2012. Experimental investigation of high frequency combustion instability in cryogenic oxygen-hydrogen rocket engines. The University of Adelaide. Ph.D. Thesis.

3. Hardi, J. S., S. K. Beinke, M. Oschwald, and B. B. Dally. 2014. Coupling of cryogenic oxygen-hydrogen flames to longitudinal and transverse acoustic instabilities. J. Propul. Power 30:991-1004.

4. Hardi, J. S., M. Oschwald, and B. Dally. 2012. Acoustic characterisation of a rectangular rocket combustor with liquid oxygen and hydrogen propellants. P. I. Mech. Eng. G-J. Aer. 227(3):436-446.

5. Hardi, J., H. Gomez Martinez, and M. Oschwald. 2014. LOx jet atomization under transverse acoustic oscillations. J. Propul. Power 30(2):337-349.

6. Schmid, P. J. 2010. Dynamic mode decomposition of numerical and experimental data. J. Fluid Mech. 656:5-28. doi: 10.1017/S0022112010001217.

7. Wierman, M., B. Pomeroy, and W. Anderson. 2013. Development of combustor response functions in a subscale high pressure transverse combustor. 5th European Conference for Aeronautics and Space Sciences (EUCASS).

8. Hardi, J., W. Hallum, C. Huang, and W. Anderson. 2014. Development of validation approaches for numerical simulation of combustion instability using flame imaging. AIAA Paper No. 2014-3775. 
9. Banuti, D. 2014. Thermodynamic analysis and numerical modeling of supercritical injection. University of Stuttgart. Ph.D. Thesis.

10. Banuti, D. T., V. Hannemann, K. Hannemann, and B. Weigand. 2016. An efficient multi-fluid-mixing model for real gas reacting flows in liquid propellant rocket engines. Combust. Flame 168:98-112.

11. Gerhold, T., V. Hannemann, and D. Schwamborn. 1999. On the validation of the DLR-TAU code. New results in numerical and experimental fluid mechanics. Notes on numerical fluid mechanics ser. 72:426-433.

12. Karl, S. 2011. Numerical investigation of a generic scramjet configuration. Technische Universität Dresden. Ph.D. Thesis.

13. Younglove, B. A. 1982. Thermophysical properties of fluids. 1. Argon, ethylene, parahydrogen, nitrogen, nitrogen trifluoride, and oxygen. J. Phys. Chem. Ref. Data 11(Suppl. 1). 353 p.

14. Lemmon, E., and R. Jacobsen. 2004. Viscosity and thermal conductivity equations for nitrogen, oxygen, argon, and air. Int. J. Thermophys. 25(1):21-69.

15. Spalart, P., and S. R. Allmaras. 1992. A one-equation turbulence model for aerodynamic flows. AIAA Paper No. 92-0439.

16. Bardina, J. E., P. G. Huang, and T. J. Coakley. 1997. Turbulence modeling validation, testing, and development. NASA. Technical Report 19970017828.

17. Wilcox, D. C. 2006. Turbulence modelling for CFD. 3rd ed. DCW Industries. 522 p.

18. Jachimowski, C. J. 1988. An analytical study of the hydrogen-air reaction mechanism with application to scramjet combustion. Hampton, VA: NASA Langley Research Center. Technical Paper 2791.

19. Lempke, M., P. Gerlinger, and M. Aigner. 2013. Assumed PDF modeling in rocket combustor simulations. Progress in propulsion physics. Eds. L. T. DeLuca, Ch. Bonnal, O. Haidn, and S. M. Frolov. EUCASS advances in aerospace sciences book ser. TORUS PRESS - EDP Sciences. 4:569-582.

20. Ivancic, B., H. Riedmann, M. Frey, O. Knab, S. Karl, and K. Hannemann. 2013. Investigation of different modeling approaches for CFD simulation of high pressure rocket combustors. 5th EUCASS. Munich.

21. Gröning, S., J. S. Hardi, D. Suslov, and M. Oschwald. 2016. Injector-driven combustion instabilities in a hydrogen/oxygen rocket combustor. J. Propul. Power 32:560573.

22. Hakim, L., A. Ruiz, T. Schmitt, M. Boileau, G. Staffelbach, S. Ducruix, B. Cuenot, and S. Candel. 2015. Large eddy simulations of multiple transcritical coaxial flames submitted to a high-frequency transverse acoustic modulation. Proc. Combust. Inst. 35(2):1461-1468.

23. Shipley, K., W. Anderson, M. Harvazinski, and V. Sankaran. 2014. A computational study of transverse combustion instability mechanisms. 50th AIAA Joint Propulsion Conference. Cleveland, $\mathrm{OH}$.

24. Schmid, M. 2014. Thermoakustische kopplungsmechanismen in flüssigkeitsraketentriebwerken. Technical University of Munich. Ph.D. Thesis. 